\title{
Center-Surround Antagonism Based on Disparity in Primate Area MT
}

\author{
David C. Bradley and Richard A. Andersen \\ Biology Division, California Institute of Technology, Pasadena, California 91225
}

Most neurons in primate visual area MT have a large, modulatory region surrounding their classically defined receptive field, or center. The velocity tuning of this "surround" is generally antagonistic to the center, making it potentially useful for detecting image discontinuities on the basis of differential motion. Because classical MT receptive fields are also disparityselective, one might expect to find disparity-based surround antagonism as well; this would provide additional information about image discontinuities. However, the effects of disparity in the MT surround have not been studied previously. We measured single-neuron responses to variable-disparity moving patterns in the MT surround while holding a central moving pattern at a fixed disparity. Of the 130 neurons tested, 84\% exhibited a modulatory surround, and in $52 \%$ of these, responses were significantly affected by disparity in the surround.
In most cases, disparity effects in the surround were antagonistic to the center; that is, neurons were generally suppressed when center and surround stimuli had the same disparity, with decreasing suppression as the center and surround stimuli became separated in depth. Also, the effects of disparity and direction were mainly additive; i.e., disparity effects were generally independent of direction, and vice versa. These results suggest that the MT center-surround apparatus provides information about image discontinuities, not only on the basis of velocity differences but on the basis of depth differences as well. This supports the hypothesis that MT surrounds have a role in image segmentation.

Key words: middle temporal area; motion perception; receptive field surrounds; image segmentation; primate; velocity tuning
The middle temporal visual area (MT or V5) is among the most widely studied cortical areas in the primate. It is functionally distinguished from other early visual areas in that its neurons are highly selective for stimulus direction and speed, and relatively insensitive to form, texture, and color (Dubner and Zeki, 1971; Zeki, 1974; Maunsell and Van Essen, 1983a,b). MT responses covary with the perception of direction in behaving monkeys (Newsome et al., 1989), and MT lesions in humans and monkeys degrade performance in tasks requiring velocity estimation (Siegel and Andersen, 1986; Newsome and Wurtz, 1988; Schiller and Lee, 1994). For these reasons MT is thought to have a central role in visual motion perception in the primate.

MT is situated on the floor and posterior bank of the superior temporal sulcus of owl and rhesus monkeys (Allman and Kaas, 1971; Dubner and Zeki, 1971; Zeki, 1974), and a functionally and anatomically similar region has been identified in humans (Tootell et al., 1995). In monkeys, MT receives much of its input from layer $4 \mathrm{~b}$ of $\mathrm{V} 1$, which has a high concentration of directionselective neurons (Dow, 1974). MT projects strongly to the medial superior temporal area, whose neurons are preponderantly directional and which appears to have a role in computing self-motion from retinal motion patterns (aki Saito et al., 1986; Bradley et al., 1996). Other projection zones of MT (ventral intraparietal area, fundus of the superior temporal sulcus) also contain directional

\footnotetext{
Received Nov. 19, 1997; revised June 30, 1998; accepted July 1, 1998.

This work was supported by grants from the National Eye Institute, the Human Frontier Science Program, and the Sloan Foundation for Theoretical Neurobiology.

We are grateful to G. Robertson, D. Ward, B. Gillikin, and S. Gertemanian for technical assistance, and to K. Shenoy for comments on this manuscript.

Correspondence should be addressed to David C. Bradley at his present address: Psychology Department, University of Chicago, 5848 S. University Avenue, Chicago, IL 60637.

Copyright (C) 1998 Society for Neuroscience $\quad 0270-6474 / 98 / 187552-14 \$ 05.00 / 0$
}

neurons (Colby et al., 1993; Desimone and Ungerleider, 1986). MT is thus situated squarely in what has been termed the visual motion pathway.

In studying the primate visual motion pathway, we have tried to consider not only how visual motion signals are measured, but also how they are used. Beyond the ability to recognize movement in the visual field, retinal motion gives other kinds of information related to structure in the environment (Wallach and O'Connell, 1953) and to our own motion with respect to the environment (Gibson, 1950). Therefore, to understand cortical motion processing, it is important to understand how motion cues are converted to other kinds of information.

One of the basic uses of motion signals is to facilitate scene segmentation (Nakayama, 1985; Braddick, 1993; Stoner and Albright, 1993). How do we know, for example, when two regions of contrast on the retina correspond to a single object or to separate objects? Although color and texture may vary over different parts of an object or surface, the direction and speed tend to be the same on all the parts. Therefore, relative motion is a useful cue for parsing an image into its separate components. This is borne out by psychophysical experiments that show that relative motion conveys a strong sense of image discontinuity (Braddick, 1993).

Several lines of evidence suggest that MT plays a role in scene segmentation and, more generally, the inference of structure from motion cues. Movshon and colleagues (1985) used stimuli composed of two surfaces (sine-wave gratings) sliding over each other to show that some MT neurons respond as if to a single surface, whereas others respond selectively to the movement of the component surfaces. This suggested that MT, as an area, is concerned not only with detecting visual motion but also with segmenting an image into its coherently and separately moving parts.

Maunsell and Van Essen (1983b) discovered that MT neurons 
are tuned for binocular disparity. They also showed that this disparity tuning does not function to compute three-dimensional motion, because none of the MT neurons studied responded selectively to motion-through-depth (instead, neurons are tuned for two-dimensional frontoparallel velocity at a particular depth). A possible explanation for the disparity tuning came from later studies from our lab, where we showed that MT neurons are normally suppressed by equidistant surfaces moving in opposite directions, but this suppression is weak or absent when the surfaces move at different depths (Bradley et al., 1995). This means that certain MT neurons can respond to the movement of a given surface without being affected by other surfaces moving at different depths. As in the studies by Movshon et al. (1985), these findings suggested that MT cells associate motion cues with a particular surface, and as such they could play a basic role in image segmentation.

The studies discussed so far were all performed in the classically defined MT receptive field, the part of the visual field in which a moving stimulus evokes a response. However, most MT receptive fields contain another region, known as the surround, which is potentially important as a mechanism for segmentation in MT. Allman and colleagues (1985a,b) first discovered this surround, a large region surrounding the classical receptive field (center), in MT of owl monkeys. Although moving stimuli in the surround do not cause MT cells to respond, their presence modulates the response to stimuli in the center. These authors showed that MT surrounds are generally antagonistic to the center; that is, cells are suppressed when surround motion has the same direction as motion in the center, but less suppressed, and sometimes facilitated, when center and surround motions are in different directions. Similarly, cells tend to respond better when stimuli in the center and surround have different speeds. These findings imply that MT firing rates carry information about differences in image motion. Allman et al. (1985a,b) proposed that center-surround interactions in MT could be used to distinguish an object's motion from its background, because motion differences tend to occur across the edges of moving objects. If so, this would support a role for MT surrounds in computations related to image segmentation.

It is possible that MT surrounds act as differential motion detectors, but that their purpose is not related to image segmentation or structure. Differential motion could be used, for example, for drawing attention to certain parts of a scene (Nakayama, 1985) or for computing self-motion during eye movements (Warren, 1995). Therefore, it is important to have independent evidence that MT surrounds play a role in image segmentation. Here we present evidence that supports such a claim. Specifically, we show that MT neurons are sensitive to disparity in the surround, and the effect of disparity in the surround is such that firing rates increase when disparity in the surround is different from the disparity in the center. This suggests that MT surrounds could be used to detect image discontinuities, not only on the basis of contrasting motions but also on the basis of contrasting depths.

\section{MATERIALS AND METHODS}

Preparation of animals. Studies were performed with two behaving, male rhesus monkeys (Macaca mulatta). Each was chronically fitted with a stainless steel head post for head immobilization, a lucite chamber for mounting an electrode microdrive, and a scleral search coil for monitoring eye position (Judge et al., 1980). Results were similar for the two animals and were pooled for the present analysis.

All procedures with animals were approved by the Caltech Institutional Animal Care and Use Committee.
Stimuli and tasks. Monkeys were seated in a dark or dimly lit room, 57 $\mathrm{cm}$ from a cathode ray tube display. The monitor was driven by a Number Nine Pepper SGT graphics board with a refresh rate of $60 \mathrm{~Hz}$, housed in an AST 386 personal computer. Stimuli were stored on a fixed disk as 1 sec (i.e., 60 frame) movies and then loaded into the memory of the graphics board before each block of trials began (see below). The same graphics system was used to generate rectangular bars for receptive field mapping.

All of our stimuli were variations of a circular random dot pattern on a dark background. The intensity of individual dots was $\sim 1$ foot lambert when viewed through colored filters (see below). Dots moved linearly at $6^{\circ} / \mathrm{sec}$ across a $4^{\circ}$ diameter circular area on the screen. When a given dot disappeared from the circle, it was "wrapped" around and reappeared at the opposite edge, thus maintaining constant dot density. To minimize flicker, all dots remained visible for the duration of the stimulus $(1 \mathrm{sec})$. All patterns contained 64 randomly positioned dots.

Each dot was represented with a pair of colored dots, one red and one green, each $0.056^{\circ}$ wide (1 pixel). These colored pairs were viewed through colored filters so that each dot was only seen by a single eye. The two members of each dot pair could then be separated horizontally to create a retinal disparity, giving the illusion of depth. When disparity was zero, a single yellow dot (i.e., a mixture of red and green) was displayed. Filters were Kodak Wratten dark red (No. 29) and dark green (No. 61). Screen intensities of the red and green colors were adjusted so that their intensities were equal when viewed through these filters. Although both filters were quite dark, crossover could not be prevented entirely; i.e., it was possible to see some red through the green filter and vice versa. However, crossover was $<10 \%$ for both filters and was clearly insufficient to disrupt the disparity tuning of MT neurons (see Results).

For each isolated neuron, three "blocks," or groups of tests, were performed using variations of the basic stimulus described above. Within a given block, all stimuli (or pairs of stimuli, as explained below) were pseudorandomly interleaved. The first two blocks measured response properties of the receptive field center. In the first block, we measured the neuron's direction tuning by showing dots moving in eight different directions $\left(0,45, \ldots, 315^{\circ}\right)$. In this test, all dots were at zero disparity. In the second block, we measured the neuron's disparity tuning by showing dots at nine different disparities $\left(-0.8,-0.6, \ldots, 0.8^{\circ}\right)$. In this block, all dots moved in the neuron's preferred direction (determined in the first block).

In the third block, we measured the effects of motion in the surround. Here, we placed one moving pattern in the receptive field center, and three patterns in the surround (Fig. 1). The center pattern moved in the neuron's preferred direction and at its preferred disparity (both defined for the center). The three surround patterns were identical to each other, but as a group had different directions (same or opposite vs center) and different disparities $\left(-0.8^{\circ}-0.8^{\circ}\right)$ on different trials. We also tested the central pattern by itself (no surround stimuli) and surround patterns by themselves (no center stimulus; see Results). These controls were interleaved with the main stimuli.

Surround patterns were placed in a triangular configuration as shown in Figure 1. The three patterns were offset vertically, with the middle pattern at the same height as the central stimulus. For a classical receptive field with radius $r$, the surround patterns were placed approximately $r$ degrees from the outer edge of the classical field. The eccentricity of the receptive fields averaged $6.2 \pm 3.4^{\circ}$ (mean $\pm \mathrm{SD}$ ), and their diameter was $7.6 \pm 2.9^{\circ}$. Surround patterns were on average $1.2 \pm 0.5 r$ units from the edge of the classical field.

All trials had the following structure. First, the monkey was given 1 sec to obtain a fixation target. The target was initially defined within a large $\left(30^{\circ}\right)$ window. After fixating the target, the monkey was given 500 msec to stabilize his gaze, after which the window collapsed down to $3^{\circ}$. The monkey was required to hold his gaze within this window for the remainder of the trial. Because only one eye position was measured, the monkey's fixation depth (vergence) could not be calculated. However, the stimuli never overlapped the fixation point, and monkeys were not required to attend to the stimuli, so it is unlikely that the disparity of the stimuli strongly affected the animals' vergence. In a recent study in which vergence was measured, fixation depth remained remarkably constant (trial-to-trial SD of $0.07^{\circ}$ ) while a monkey viewed, and in that experiment attended to, similar stimuli at similar eccentricities (Bradley et al., 1998).

After the 500 msec gaze stabilization period, the monkey continued to fixate for another $3200 \mathrm{msec}$, during which time two visual stimuli were shown. This first stimulus lasted $1 \mathrm{sec}$, followed by a $1 \mathrm{sec}$ pause and then 


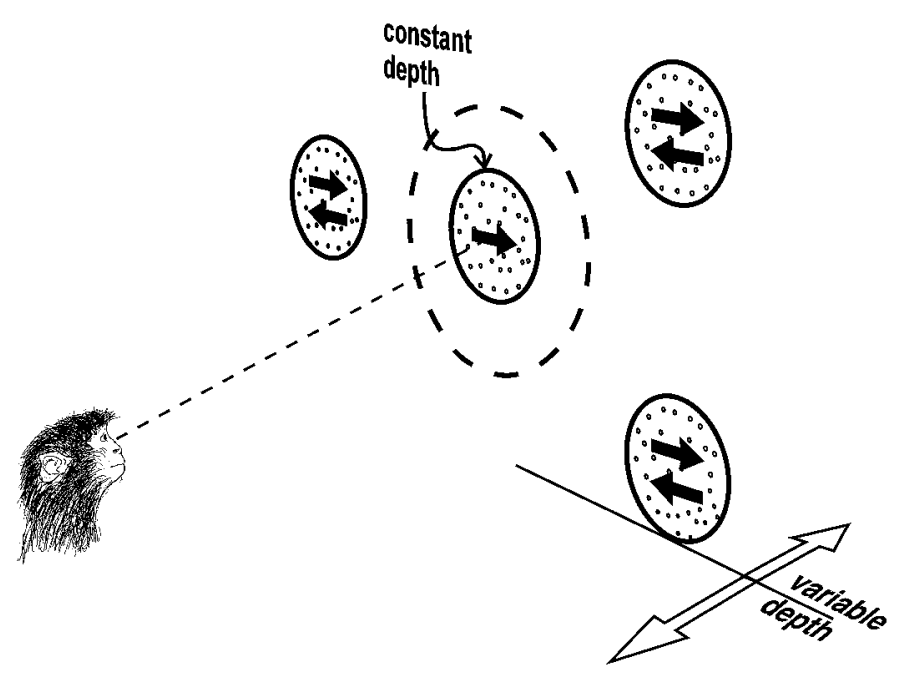

Figure 1. Disparity effects in the MT surround were measured by placing a moving random dot pattern in the receptive field center and three moving patterns in the surround. The central pattern was always the same, moving in the preferred direction and at the preferred disparity (as defined for the center). The opposing arrows in the surround patterns are meant to indicate that these patterns moved either in the same direction as the center, or in the opposite direction, on different trials (but not simultaneously in both directions). Also, surround patterns were shown at different disparities on different trials. All three surround patterns were identical to each other; i.e., on a given trial they moved in the same direction and had the same disparity (so they appeared to be in the same depth plane).

another $1 \mathrm{sec}$ stimulus. The purpose of this two-stimulus design was simply to maximize data collection for a given number of trials.

For testing direction selectivity, opposite directions were shown in the two stimulus segments of a given trial. For testing disparity selectivity, adjacent disparity classes were shown (e.g., $-0.8^{\circ},-0.6^{\circ}$ ). Thus, although stimulus pairs were pseudorandomly interleaved, the sequence of stimulus segments within a given pair was fixed (because of software limitations), and this could create an ordering effect within each pair. Therefore, in 110 cells, we reassessed direction tuning by showing the preferred-antipreferred direction pair in random order. Indices of directionality (see below) were the same, whether the sequence was preferred then antipreferred or antipreferred then preferred (median 0.98 and 0.98 , respectively; $n=110$ ), suggesting that the order of stimuli within a pair did not strongly affect direction tuning.

Similar controls were not performed for disparity tuning. However, because adjacent disparity levels were shown in each pair, an ordering effect-if it occurred-would mainly affect the relative responses within each pair, without substantially changing the overall shape of the disparity tuning curve. Because MT neurons are broadly tuned for disparity (Maunsell and Van Essen, 1983b), it is mainly this general shape that concerns us.

For testing surround effects, the two stimulus segments for a given trial consisted of opposite directions in the surround. In this case, however, the order of stimuli within each pair was randomized for the majority of cells $(110 / 130)$, and data corresponding to opposite-order pairs were pooled to average out potential order effects. Also, data from all cells $(n=130)$ were analyzed with a two-way ANOVA, which accounted for direction and disparity (opposite orders pooled), and a second analysis was performed for the random-order subset $(n=110)$ with a three-way ANOVA, which accounted for direction, disparity, and order (as well as all first-order interactions). The results pertaining to direction and disparity effects, and the interaction between them, were the same in both cases (see Tables 1,3), so opposite-order trials were pooled and not treated further in this analysis.

Recording procedure. Neurons were accessed on long, dorsoventral penetrations with tungsten microelectrodes. The electrodes were advanced with a Fred Haer (FHC) chronic microdrive system and advanced through stainless steel guide tubes that penetrated the dura. Area MT was generally located $\sim 10 \mathrm{~mm}$ beneath the cortical surface and identified on the basis of physiological properties (e.g., direction tuning, disparity tuning, direction opponency), receptive field size, and consistent topographical organization.

When a cell was isolated, its receptive field was mapped by dragging a bar or dot pattern around the screen with a digital mouse while the monkey performed a fixation task. Cell activity was monitored on-line by amplifying electrode potentials on a portable stereo. When collecting data, a window discriminator was used to generate a transistor to transistor logic (TTL) impulse for each action potential detected, and the time of this impulse was recorded on a digital computer. Cell firing rates could thus be assessed by counting the number of spikes registered within a given time interval. Data were collected with an 80486-based personal computer. This data collection computer was also used to monitor eye position, administer rewards for successful trials, and send commands to the graphics computer (see above) to start and stop stimulus presentation.

General data analysis. Neural responses were expressed as the mean firing rate (spikes/second) over the last $800 \mathrm{msec}$ of the $1 \mathrm{sec}$ stimulus periods. These raw responses were adjusted for the baseline activity (of a given cell) by subtracting the firing rate that occurred in the absence of any stimulus (background). All calculations were based on these baseline-adjusted firing rates.

Most of our statistical tests were performed in terms of a generalized linear model (GLM), which handles ANOVA and regression problems within the framework of linear least-squares regression (Fox, 1997). For regression problems (quantitative independent variables), the GLM works like a conventional linear regression, but for ANOVA (discrete independent variables), the GLM uses "dummy" variables ( 0 or 1$)$ to allow discrete effects to be included in the linear regression equation. The main advantage of GLM over conventional ANOVA and regression techniques is that GLM allows tests of "mixed" models, which include both quantitative and discrete independent variables.

Several implementations of the GLM were used to test hypotheses about the data. To test the effects of disparity in the receptive field center, the GLM was configured as a one-way ANOVA, which models responses as a function of a single, discrete variable (disparity). To test the simultaneous effects of direction and disparity in the surround, we used a two-way ANOVA, with direction and disparity as the discrete independent variables.

A third form of the GLM was used to compare the shapes of disparitytuning curves. For example, to compare disparity tuning in the receptive field center with disparity tuning in the surround, we plotted the response to a particular disparity in the center versus the response to the same disparity in the surround (this was possible because the same disparity values were tested in the center and the surround). A positive slope for this relationship implies that the two curves have similar shapes, whereas a negative slope implies opposite (inverted) shapes (see Fig. 8). This slope was calculated with a GLM configured as a simple linear regression, where the dependent variable was the response to a particular disparity in the surround, and the (quantitative) independent variable was the response to the same disparity in the center. This simple regression was performed either by itself or as part of a mixed-model GLM, which also included a discrete variable to represent the effect of direction (thus one quantitative and one discrete independent variable). Similar models were used to compare disparity tuning curves for opposite directions in the surround. All GLM methods used in this analysis are explained in detail in the Appendix.

To identify instances where the response to a specific stimulus (i.e., to a given direction and disparity in the surround) was significantly higher or lower than the response to the center pattern by itself, we performed individual $t$ tests between the center-alone response and the responses to all 18 surround stimuli. Because repeated $t$ tests collectively have a high false-positive error rate, we performed them only on neurons showing significant surround effects in the two-way ANOVA treatment discussed above. In other words, by limiting ourselves to neurons shown by ANOVA to have significant surround effects, we contain the high falsepositive rate associated with multiple $t$ tests, without having to use low $p$ values on the individual tests, which decreases their power (Fisher's protected $t$ test) (Carmer and Swanson, 1973).

To quantify the strength of direction tuning in a given neuron, we calculated the "direction index," defined as $D_{\mathrm{I}}=(P-A P) /(P+A P)$, where $P$ is the response to motion in the preferred direction, and $A P$ is the response to motion in the antipreferred (opposite) direction (Snowden et al., 1991). $D_{\mathrm{I}}=0$ means the response was the same for preferred and antipreferred directions, $D_{\mathrm{I}}=1$ means no response to the antipre- 


\section{example}

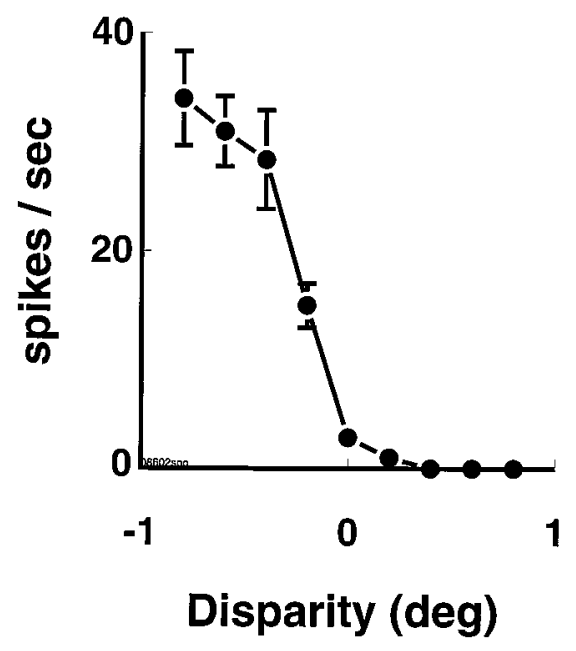

population

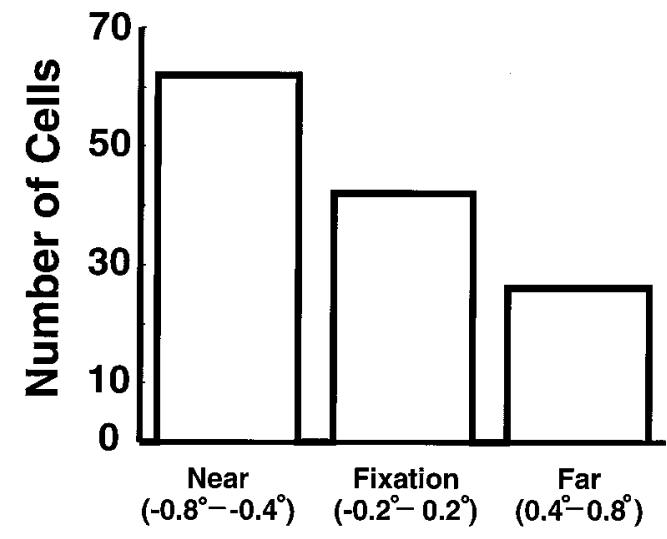

Preferred Disparity
Figure 2. Disparity effects in the classical MT receptive field. The left panel shows an example of disparity tuning in an MT neuron. The points and error bars are means \pm SE. The firing rate was strong at negative (near) disparities, and in this example, nil at positive (far) disparities. The right panel shows the distribution of preferred disparities for the entire neuron sample ( $n=$ 127). Neurons were classified as neartuned, far-tuned, or fixation-tuned, depending on the disparity at which the peak response occurred (see Results). The distribution shows that near-tuned cells were more common than fixation-tuned or fartuned cells. ferred direction, and $D_{\mathrm{I}}>1$ means the response was actually suppressed (below baseline) for the antipreferred direction.

An index of disparity tuning strength was also calculated; in this case we used the maximum and minimum responses from the disparity tuning curve, and the index was $(\max -\min ) /(\max +\min )$.

Significance tests were performed at the $\alpha=0.05$ level. All \pm symbols refer to SE, except where stated otherwise.

\section{RESULTS}

A total of $130 \mathrm{MT}$ neurons from two monkeys were tested for disparity tuning in the surround. All of these were also tested for direction and disparity tuning in the center (see below), but in a few instances the center-disparity tuning data were not saved. The sample sizes for the various calculations below thus range from 127 to 130 neurons.

\section{Direction and disparity effects in the receptive field center}

After isolating each neuron and mapping its receptive field, we centered a random dot pattern in the receptive field and measured responses to various directions of motion. Most of the isolated cells showed a strong preference for a particular direction, as is characteristic of MT (Dubner and Zeki, 1971; Zeki, 1974; Maunsell and Van Essen, 1983a). This is called the "preferred" direction, and the opposite direction is called "antipreferred." The strength of the neurons' direction preference is reflected in the direction index (see Materials and Methods), whose median was 0.97 in the sample population $(n=130)$. The proximity of this value to 1 means that responses to the antipreferred direction were generally small compared with responses to the preferred direction.

Most of the neurons studied were also sensitive to disparity in the receptive field center, confirming earlier reports (Maunsell and Van Essen, 1983b; Bradley et al., 1995). This was demonstrated by showing a preferred-direction pattern at nine different disparities $\left(-0.8^{\circ}\right.$ to $\left.0.8^{\circ}\right)$. The majority of the cells in our sample $(82 \%)$ responded differently to different disparities $(p<0.05$, one-way ANOVA). An index of disparity tuning strength was calculated analogously to the direction index (see Materials and Methods). The median of this index was 0.38 in the sample as a whole $(n=127)$.

Figure 2 shows the distribution of neurons according to the preferred depth of their receptive field center. Neurons were classified as near-tuned if their peak response occurred at disparities between $-0.8^{\circ}$ and $-0.4^{\circ}$, far-tuned for peaks between $0.4^{\circ}$ and $0.8^{\circ}$, and fixation-tuned (i.e., preferring depths near the fixation point) for peaks between $-0.2^{\circ}$ and $0.2^{\circ}$. The distribution of these three types shows a decreasing frequency going from near-tuned to far-tuned (left to right on the graph). Therefore, there is a bias in macaque MT for stimuli appearing in the foreground. We will see below that the opposite bias exists in the surround.

\section{Placement of surround stimuli}

The central (classical) receptive field of each neuron was mapped by dragging a bar or dot pattern around the screen to delimit the region in which the neuron reacted to the stimulus. Surround stimuli were subsequently placed outside this region, at distance of $\sim r$ degrees from the edge of the classical field, where $r$ is the estimated radius of the classical field. To be certain that surround stimuli were outside the central field, we measured responses to surround patterns in the absence of a central stimulus. Mean responses for all cells were $5 \pm 1$ and $1 \pm 1$ spikes/sec when the surround patterns moved in the center's preferred and antipreferred direction, respectively. For comparison, mean responses to a central pattern by itself were $57 \pm 4$ and $4 \pm 2$ spikes/sec, respectively, for the preferred and antipreferred direction. This suggests that surround patterns were not always completely outside the classical field, because weak responses were obtained and direction preferences tended to resemble those in the center. However, the analyses below clearly show that stimuli in this "surround" region strongly suppress responses to central stimuli, and this suppression is strongest when the surround patterns move in the center's preferred direction (i.e., responses are higher when surround patterns go in the center's antipreferred direction), leaving little doubt that the surround patterns were in an antagonistic region. These findings suggest that the classical field and the antagonistic surround overlap somewhat.

\section{Disparity tuning in the MT surround}

Disparity tuning in the MT surround was studied, in a given cell, by holding a central pattern at the neuron's preferred disparity while varying the disparity of stimuli in the surround (Fig. 1). This is analogous to the way disparity tuning was measured in the 
example

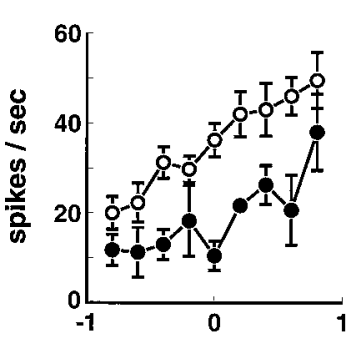

Surround Disparity (deg)

\section{population}

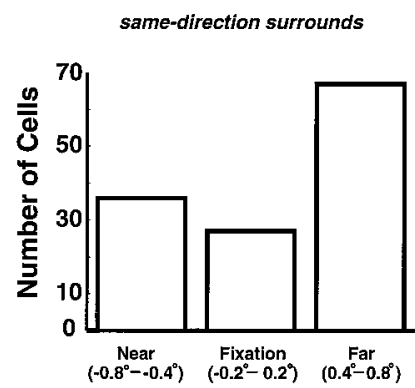

opposite-direction surrounds

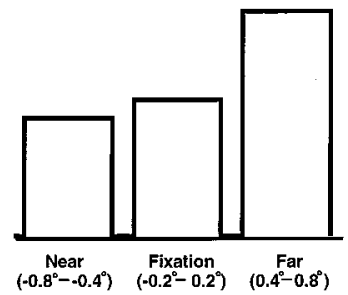

Preferred Surround Disparity

Figure 3. Disparity effects in the receptive field surround. The left panel shows an example based on a single MT neuron. Points and error bars are means \pm SE. The two tuning curves were generated while holding a central pattern at a fixed disparity (the preferred disparity for the center). Closed circles show data for surround patterns moving in the same direction as the center pattern; open circles indicate opposite-direction surrounds. For both directions, firing rates increase as surround disparities go from negative to positive (near to far). However, firing rates were higher overall for opposite-direction surrounds, as was typically the case. The right panels show the distribution of preferred disparities in the surround. For both sameand opposite-direction surrounds, the cells tend to prefer far disparities.

center, except that surround patterns were shown moving in two different directions (same or opposite relative to center). Therefore, two disparity tuning curves were generated for each MT surround. Recall that the center pattern was always shown with the preferred direction and disparity (as defined for the center).

Figure 3 shows the distribution of preferred depths in the surround. As with our analysis of the center (see above), cells were classified as near-, far-, or fixation-tuned, judging from the effects of surround disparity on the center response. The distribution of the three tuning types (Fig. 3) shows an increasing prevalence going from near- to far-tuned; i.e., the most common cell responded best to far disparities in the surround. This implies that MT neurons tend to prefer background (far) motion in the surround. This is opposite to the center, where foreground (near) motion typically causes the strongest response (Fig. 2). We will show below that this dichotomy stems from a more general phenomenon, which is that disparity effects in the center and the surround are usually opposite; that is, the disparities that cause the strongest excitation in the center tend to cause the strongest suppression in the surround.

\section{Simultaneous analysis of direction and disparity effects in the MT surround}

Because the various combinations of direction and disparity in the surround were interleaved, the effects of direction and disparity could be evaluated simultaneously with a two-way ANOVA. This analysis, implemented under the GLM, is described in the Appendix and illustrated schematically in Figure 5.

Briefly, the ANOVA tries to explain the response to a given stimulus in the surround as the sum of four separate effects, or parameters: (1) the overall effect, which is the mean response for all stimuli in the surround, plus (2) the specific effect of the surround stimulus's direction, plus (3) the specific effect of the surround stimulus's disparity, plus (4) the "conditional" effect, which is the interaction between direction and disparity. These effects were tested separately with incremental $F$ tests $(\alpha=0.05)$, allowing us to assign each cell to one of five categories.

Cells with additive direction and disparity effects. In many cells, responses were significantly affected by both direction and disparity in the surround, and there was no significant interaction.
This implies that the direction and disparity effects were parallel, or additive.

Figure $4 A$ shows an example of a neuron with additive direction and disparity effects. In this and the other panels of the figure, the points and error bars represent the responses to various directions and disparities in the surround, and the dashed horizontal line represents the response to the central pattern by itself. Each panel shows two disparity-tuning curves, one for each direction. The shift between the curves is the effect of direction, whereas the span of the curves represents the effect of disparity (note that the model does not assume a linear relationship between the response and the independent variables, direction and disparity; however, it is linear with respect to its parameters or effects).

In "additive" cells such as the one shown in Figure $4 A$, the effect of disparity is the same for both directions, and-equivalently - the effect of direction is the same for all disparities. Graphically, this means that the two disparity-tuning curves have the same shape (constant disparity effect) and that the curves are offset by a constant amount (constant direction effect). The two effects are thus additive, and the difference between the lowest point on the lower curve and the highest point on the higher curve represents the total effect of direction and disparity. Figure 5 illustrates this principle.

Thirty of $130(23 \%)$ of the neurons showed significant, additive direction and disparity effects. For these cells, it is useful to compute the total response range associated with direction and disparity, because this range can be decomposed into the range attributable to direction plus the range attributable to disparity. For this calculation, we normalized responses from each cell to the overall mean response (across all surround stimuli) for that cell. In the following, effect magnitudes are reported as percentages of this overall mean.

The effect of direction averaged $52 \pm 10 \%$; that is, surround disparity-tuning curves were offset by $52 \%$, on average, as a function of different directions in the surround. In the majority of cases $(23 / 30 ; 77 \%)$, this offset was such that responses to oppositedirection surrounds (relative to center) were higher than responses to same-direction surrounds (e.g., Fig. 4A). The effect of disparity, represented by the span of each disparity-tuning curve 


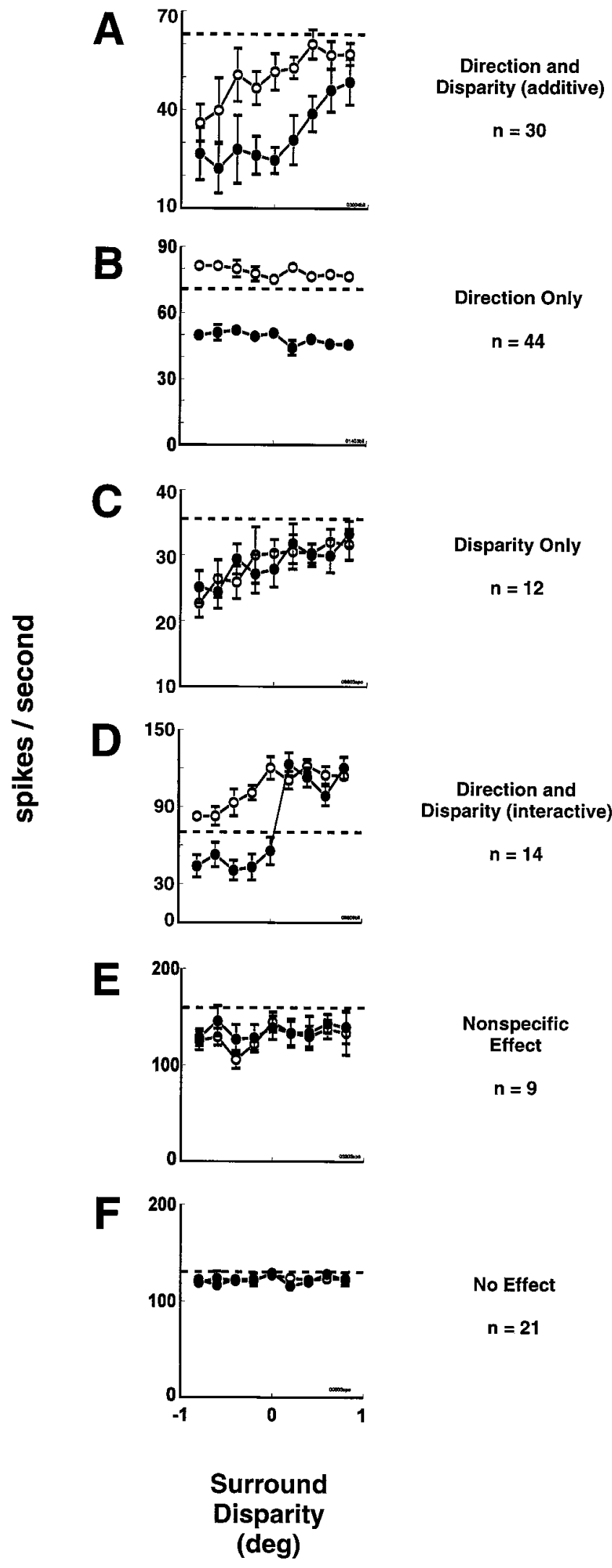

Figure 4. Examples of the different cell types, as classified by two-way ANOVA. Each panel shows data from a representative cell. In all panels, points and error bars represent means $\pm \mathrm{SE}$, and the dashed horizontal line represents the mean response to the central pattern by itself. Closed circles represent surround patterns moving in the same direction as the central

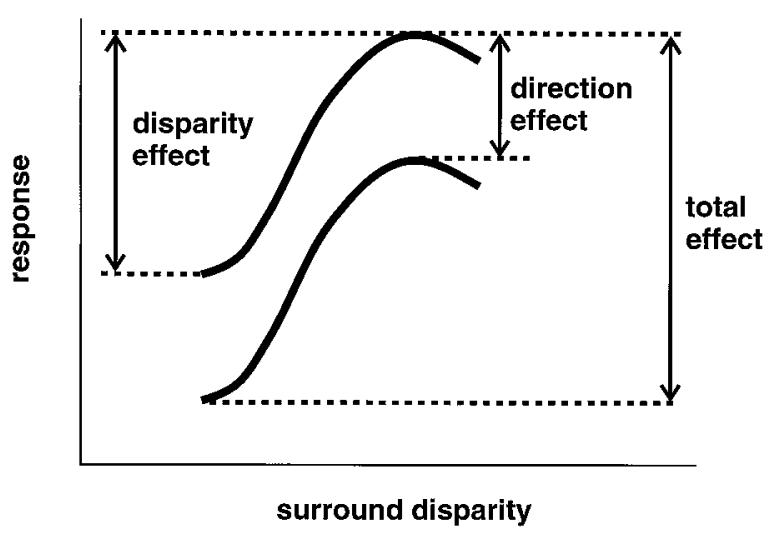

Figure 5. Schematic showing how two-way ANOVA tries to explain responses by adding the effects of direction and disparity. Each curve represents the disparity tuning profile for a given direction (same or opposite with respect to the central pattern). The disparity effect is seen as the range of responses going from the base to the peak of a given tuning curve, and the direction effect is seen as the vertical offset between the curves. In this example, it is assumed that there is no interaction; i.e., the two curves are parallel. In the absence of an interaction, the total effect is simply the sum of the direction and disparity effects.

(recall that the two curves are identical), averaged $67 \pm 20 \%$. The combined effect of direction and disparity averaged $119 \%$; that is, for a hypothetical neuron with mean firing $=100$, the response varied from 41 to 160 , on average, as a result of changing direction and disparity in the surround.

The range estimates provide an intuitive measure of the size of direction and disparity effects. However, because nine disparity values were tested, compared with only two directions, the range attributable to disparity tends to be inflated. For comparative purposes, a more appropriate measure is $R^{2}$, the portion of variance attributable to each factor, which does not depend on the number of values tested. Direction and disparity had comparable $R^{2}(0.25 \pm 0.04$ and $0.18 \pm 0.02$, respectively), accounting for roughly one-fifth to one-quarter of the variance each (see Table 2).

Cells with only direction effects. Forty-four of 130 (34\%) of the cells showed significant effects of direction in the surround, but no effect of disparity, and no direction-disparity interaction. Figure $4 B$ shows an example. The disparity-tuning curves are flat (no disparity effect), but they are offset by the effect of direction. The $R^{2}$ of the direction effect in these cells averaged $0.22 \pm 0.03$ (Table 2).

Cells with only disparity effects. A small number of cells (12/130;

$\leftarrow$

pattern; open circles represent opposite-direction surrounds. Baseline firing rates were not subtracted before graphing the data. $A$, An additive effect, where direction and disparity both influence the firing rate, but each effect is largely independent of the other. $B$, Only a direction effect. The opposite-direction surrounds produce a higher response than samedirection surrounds, but there is no appreciable effect of disparity; i.e., the curves are flat. $C$, Only a disparity effect. For both surround directions, responses increase as disparities go from negative to positive, but there is no significant offset between curves representing same- and oppositedirection surrounds. $D$, An interactive effect. Both direction and disparity affect the firing rate, but the magnitude of the direction effect depends on disparity and vice versa. $E$, A nonspecific effect. Responses are suppressed, overall, compared with the response to the central pattern by itself (dashed horizontal line), but responses are roughly constant for different directions and disparities. $F$, No effect. All responses, regardless of direction or disparity, are roughly equal to the response to the central pattern by itself. 
9\%) showed significant effects of disparity, but no effect of direction and no direction-disparity interaction. Figure $4 C$ shows an example. In this case, the two disparity-tuning curves are roughly superimposed, implying no effect of direction, but both curves span an appreciable response range. The $R^{2}$ of the disparity effect in these cells averaged $0.20 \pm 0.01$ (Table 2).

Cells with interacting direction and disparity effects. Only 14/130 $(11 \%)$ of the cells showed a significant interaction between direction and disparity effects; that is, both direction and disparity affected the firing rate, but the effect of one depended on the other. Figure $4 D$ shows an example. That the two disparitytuning curves are not parallel means the effect of direction was different for different disparities. By the same token, the different shape of the two disparity-tuning curves means the effect of disparity was different for different directions. In this example, the effect of direction was strongest at negative disparities. The mean $R^{2}$ of the interaction effect was $0.13 \pm 0.02$; i.e., approximately half the values for direction and disparity "main" effects (see above).

When direction and disparity effects interact, it is pertinent to ask whether the effects simply change in magnitude or whether they actually reverse. For example, if the two disparity-tuning curves were to cross each other, this would mean that direction had opposite effects at different disparities (on either side of the crossover point). Figure 6 (top) illustrates this point. For each "interaction" cell, we compared the responses to the two directions in the surround ( $t$ test, $p<0.05$ ) for each value of disparity (i.e., one $t$ test of same vs. opposite direction for each of the nine disparities). Only one neuron showed both a significantly positive difference and a significantly negative difference, implying that the direction effect had reversed at different disparities. All of the remaining 13 cells also showed significant differences $(4 \pm 1$ significant differences on average), but in a given cell these differences were always of the same polarity $(+$ or -$)$. Therefore, even when direction and disparity interact, the direction effect changes quantitatively but not qualitatively; that is, it changes in magnitude but (with one exception) does not reverse.

It is also possible that disparity effects could reverse at different directions. Graphically, this would translate into changes in the disparity-tuning curve for one direction being mirrored by opposite changes in the disparity-tuning curve for the other direction (Fig. 6, bottom). As explained in Materials and Methods, we can test for this by plotting one curve as a function of the other (see Fig. 8). The slope of this relationship (calculated by regression) tends to be negative when the curves have opposite shapes and positive when the curves have similar shapes. Only 4 of the 14 cells with interaction showed a significant relationship (slope) between the two disparity-tuning curves, and only two of the four significant slopes were negative. Therefore, there was no consistent reversal of the disparity effect for different directions in the surround.

When an interaction exists, it is meaningless to calculate "the" magnitude of the direction and disparity effects, because these effects are not constant. However, it is important to understand that by virtue of the direction-disparity interaction, these cells were necessarily affected by both direction and disparity in the surround.

Cells with nonspecific surround effects. In a small number of cells (9/130), responses were unaffected by surround direction or disparity (or their interaction), but the mean response was nevertheless significantly different in the presence of surround stimuli compared with their absence (mean of all surround responses

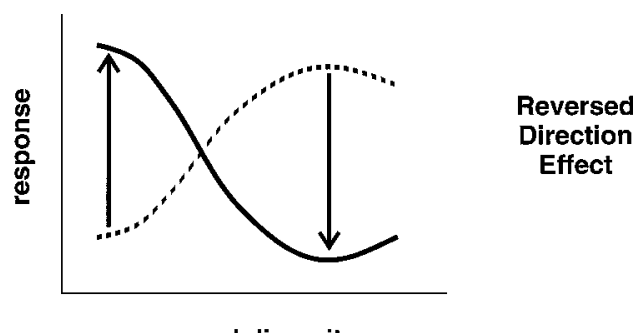

surround disparity

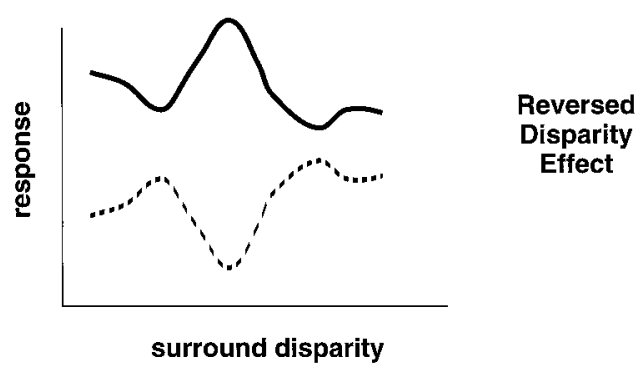

Figure 6. Schematic showing how direction effects could reverse at different disparities (top), and how disparity effects could reverse for different directions (bottom). For a disparity reversal, we would see increases in one curve associated with decreases in the other curve and vice versa. For a direction reversal, the order of responses corresponding to the two surround directions would be opposite at different disparities.

tested against the mean response to the center pattern alone). These cells were thus sensitive to surround motion without being specifically affected by the direction or disparity of the surround stimulus. Figure $4 E$ shows an example.

Cells with no surround effect. The remaining 21/130 cells (16\%) were unaffected by stimuli in the surround, regardless of their direction or disparity (all tests described above were nonsignificant). These cells are tentatively classified as not having a sur-

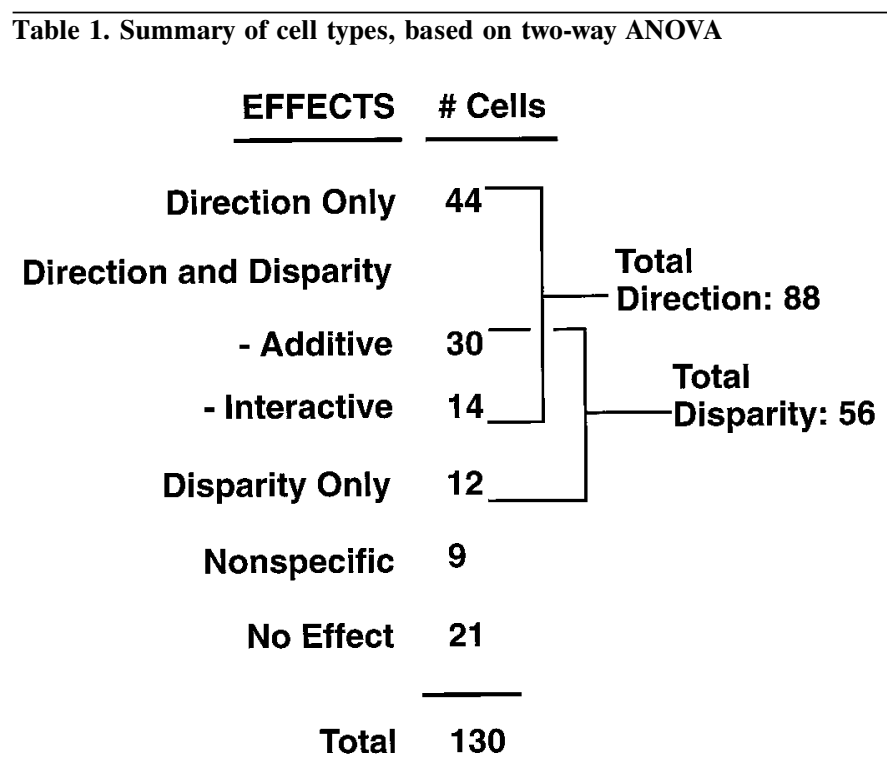

A total of 88 cells showed significant direction effects, either alone or in combination with a disparity effect (additive or interactive). Similarly, 56 cells showed disparity effects, either alone or in combination with a direction effect. The ANOVA was computed in a regression framework (general linear model) with both independent variables treated as nominal-scale (discrete). 


Table 2. Summary of $\boldsymbol{R}^{\mathbf{2}}$ values for the different cell types (see Table 1)
\begin{tabular}{llll} 
Mean $\pm R^{2}$ & & \\
\cline { 2 - 4 } Cell type & Direction effect & Disparity effect & Direction-Disparity \\
\hline $\begin{array}{l}\text { Additive } \\
\text { Direction only }\end{array}$ & $0.25 \pm 0.04$ & $0.18 \pm 0.02$ & \\
$\begin{array}{l}\text { Disparity only } \\
\text { Interactive }\end{array}$ & & $0.20 \pm 0.01$ & $0.13 \pm 0.02$ \\
\hline
\end{tabular}

Each value shown is the mean for that group of cells \pm SEM.

\begin{tabular}{lll}
\hline Table 3. Summary of three-way ANOVA results \\
& $\begin{array}{l}\text { Number } \\
\text { significant } \\
(p \leq 0.05)\end{array}$ & \\
Factor & 74 & 6110 cells \\
\hline Direction & 47 & $43 \%$ \\
Disparity & 58 & $53 \%$ \\
Stimulus order & 14 & $13 \%$ \\
Direction by disparity & 18 & $16 \%$ \\
Direction by stimulus order & 4 & $3.6 \%$ \\
Disparity by stimulus order & 4 &
\end{tabular}

This analysis included six independent variables, representing direction, disparity, and stimulus order, and the interactions direction-by-disparity, direction-by-order, and disparity-by-order. The ANOVA was computed in a regression framework (general linear model) with all independent variables treated as nominal scale (discrete).

round, although it is possible that they did have surrounds but effects were too small or variable to detect.

\section{Summary of two-way ANOVA results}

Table 1 summarizes the ANOVA results. Overall, responses in $109 / 130$ cells $(84 \%)$ depended significantly on the type and/or presence of stimuli in the surround. Among these, 56/109 (52\%) were significantly affected by the disparity of surround stimuli (with or without a concomitant effect of direction). Similarly, $88 / 109(81 \%)$ were significantly affected by direction in the surround (with or without a concomitant effect of disparity). Thus, most cells (100/109; 92\%) showing any surround effect were sensitive to direction or disparity or both in the surround.

Many cells (44/109; 40\%) showed both direction and disparity effects. In most of these (30/44), the two effects were additive. In a smaller number (14/44), the effects interacted; that is, the effect of direction depended on disparity, and vice versa. However, even in cells with significant interactions, there was little evidence that either effect reversed, and the interaction effects accounted for a relatively small portion of the total variance (Table 2 ). The low occurrence and magnitude of interaction effects suggest that the effects of direction and disparity in the MT surround for the most part are independent.

Recall that two stimuli, with identical disparities but opposite directions, were shown on each trial, and in most cells $(110 / 130)$ the order of the two directions was randomized to cancel potential stimulus-order effects. However, we also performed a threeway ANOVA in which the order of the two directions in each pair was taken into account, rather than pooled as in the preceding analysis. Approximately one-half of the cells showed a significant order effect (Table 3), such that the second response was on average $15 \pm 6 \%$ greater than the first. The significance of this is unclear. However, the results pertaining to direction and disparity were unchanged; that is, the percentages of cells showing

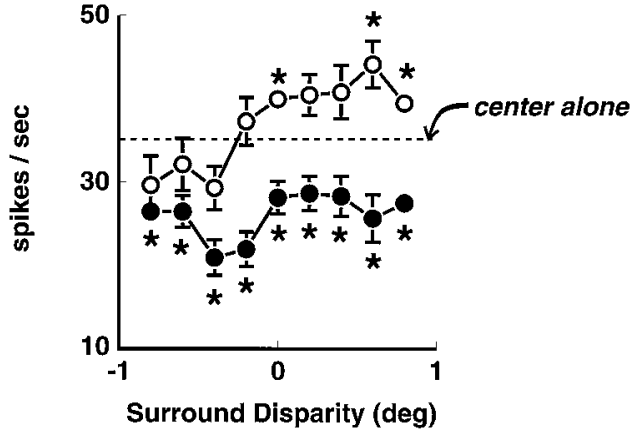

Figure 7. Example of specific suppressive and excitatory effects in a given neuron. Points and error bars represent mean responses $\pm \mathrm{SE}$, and the dashed horizontal line represents the mean response to the central pattern by itself. Each asterisk represents a significant difference $(p<$ $0.05, t$ test) between the response in question and the response to the central pattern alone. For same-direction surrounds (solid circles), all nine responses were significantly suppressed. For opposite-direction surrounds, three of the nine responses were significantly facilitated.

additive, interactive, direction-only, and disparity-only effects (Table 3) were nearly identical to the percentages reported for pooled data (Table 1). This is not surprising given that in most cells $(110 / 130)$ stimulus order was orthogonal to direction and disparity; that is, both stimulus orders were tested for every combination of direction and disparity. This means that although the two-way ANOVA did not take stimulus order into account (as opposed to the three-way, which did), the estimated effects of direction and disparity still could not have been biased, because potential order effects (including interactions with direction and disparity) were forced to average out. On the other hand, to the extent that stimulus order did have an effect, excluding it from the model (as in the two-way ANOVA) could decrease statistical power by increasing the amount of unexplained variance. However, given the similarity of the two- and three-way results, the inclusion or exclusion of stimulus order from the ANOVA did not make a substantial difference.

\section{Suppression versus facilitation}

The ANOVA discussed above determines which cells are affected by movement in the surround and how much they are affected. In this section we determine how often surround stimuli caused responses to increase (excitatory effects) or decrease (suppressive effects) in relation to the response to a central stimulus by itself.

In the 109 neurons showing significant surround effects in the two-way ANOVA, we $t$ tested individual responses to different stimuli in the surround against the response to a center pattern by itself (Fig. 7). In 85 of these (78\%), the response was significantly lower, for at least one of the surround stimuli, than the response to the center pattern alone. Only in 30 cells $(28 \%)$ was there at least one response significantly higher than the response to the center alone. Therefore, surround effects in MT were generally suppressive.

This preponderance of suppressive effects over excitatory effects was true for both same- and opposite-direction surrounds, but it was most pronounced for same-direction surrounds. Thus, taking data separately for same- and opposite-direction surrounds, the ratio of suppressive to excitatory effects was greater for same-direction surrounds (75:11, i.e., 7:1) than for oppositedirection surrounds $(51: 25$, i.e., $\sim 2: 1)$. Therefore, surround patterns in general were more likely to suppress than excite, and they 

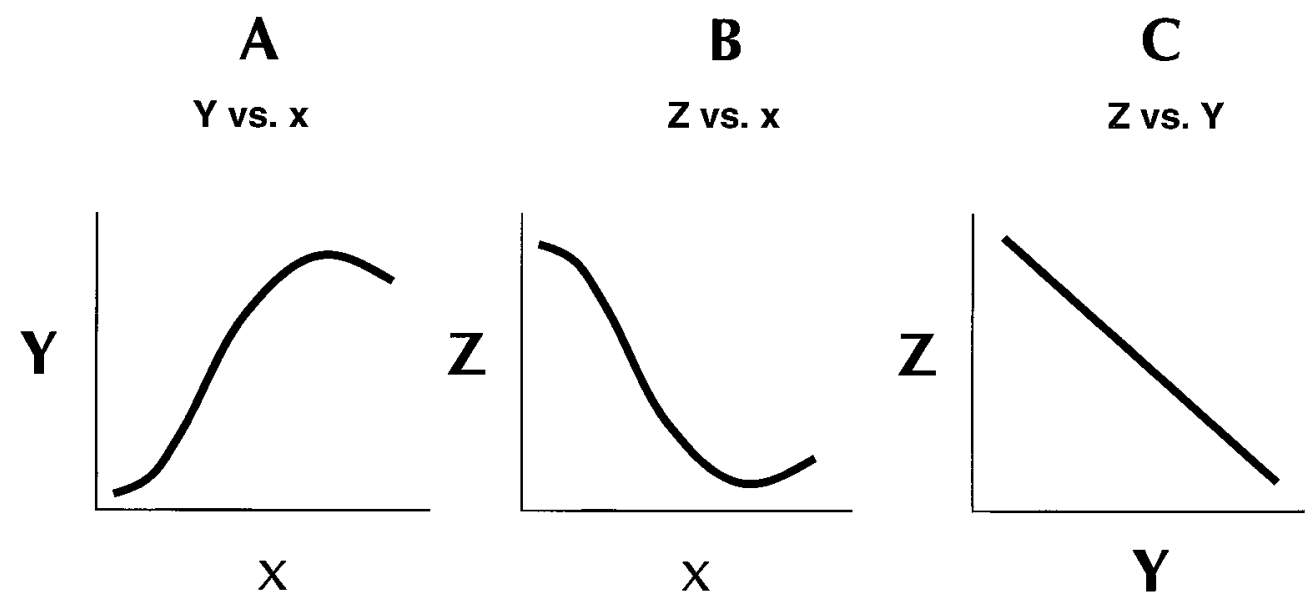

Figure 8. Schematic illustrating how regression can be used to compare the shapes of disparity tuning curves. $A$ and $B$ represent disparity tuning curves under two different conditions. $C$ plots the responses from $B$ (ordinate) against the responses from $A$ (abscissa). Because the curves in $A$ and $B$ have vertically opposite (inverted) shapes, the slope in $C$ is negative. If instead their shapes were similar, the slope in $C$ would be positive. $x$ is disparity, $Y$ is the response to a given disparity in the center, and $Z$ is the response to a given disparity in the surround. Because $Y=f(x)(A)$ and $Z=f(x)(B)$ were measured at the same $x$ values (disparities), $Z=f(Y)$, the relationship between center and surround tuning $(C)$ is known for the measured set of $x$ values.

were particularly likely to suppress (and less likely to excite) when center and surround motion occurred in the same direction.

\section{Center versus surround disparity effects}

In the preceding sections we discussed the effects of direction and disparity in the surround and compared responses to a central stimulus with and without stimuli in the surround. In this section we compare disparity effects between the center and the surround.

The basic analysis is illustrated in Figure 8. Because the same nine disparities were used to measure disparity tuning in the center and the surround, we can plot, for a given cell, the mean response to each disparity in the center versus the mean response to the same disparity in the surround (recall that surround disparity tuning is measured with the central pattern held at a fixed disparity). If the slope of this relationship is positive, the two disparity-tuning curves must have similar shapes. On the other hand, if the slope is negative, the two curve shapes are inverted, or vertically mirror-imaged. If the slope is insignificant (neither positive nor negative), neither conclusion is drawn.

For a given cell, the slope of the relationship between center and surround disparity tuning was calculated for each of the two surround directions. These slopes were calculated independently, but simultaneously, with a GLM configured as a multiple regression that includes a dummy variable to represent direction. This model is detailed in the Appendix.

Briefly, the model calculates the linear relationship, or slope, between disparity tuning in the center and disparity tuning in the surround, once for same-direction surrounds and once for opposite-direction surrounds (recall that "same" and "opposite" are relative to center). Although the slopes are calculated independently for the two surround directions, the model also computes a direction-slope interaction term that tells whether the two slopes are different for the different surround directions. (Note that in the previous section we compared disparity-tuning curves between different directions in the surround, whereas here we are comparing disparity-tuning curves between the center and the surround.)

In this analysis, we are interested in (1) the slope of the relationship between center and surround disparity tuning and
(2) how that slope differs for opposite directions in the surround. The results of the multiple regression thus allow the following classification (note that this classification is separate from the classification described in the previous section).

Cells with opposite center and surround disparity tuning. Disparity tuning data for both the center and surround were collected in 127 cells. In $30 / 127$ of these (24\%), there was a significant, negative relationship between the disparity tuning in the center and the disparity tuning in the surround. Figure $9 A-C$ shows an example. Figure $9 A$ shows the disparity tuning for the center of the receptive field (i.e., no surround stimuli). The neuron clearly responded best to negative (near) disparities. Figure $9 B$ shows the same neuron's disparity tuning in the surround. Here, the disparity of the central pattern was held at $-0.8^{\circ}$ (the preferred disparity for the center), whereas the disparity of the patterns in the surround was varied. The two curves represent opposite directions in the surround. Both curves suggest that the neuron responded best when the surround patterns were at positive disparities. The relationship between center and surround disparity tuning is shown in Figure $9 C$. Each of the slanted lines (one for each surround direction) represents the regression fit to the data (points are the actual data). The negative slope of these relationships means that disparity effects were opposite in the center and the surround; that is, the disparities that caused the greatest excitation in the center tended to cause the greatest suppression in the surround. This was true for both surround directions.

Neurons in this category did not show a difference between the regression slopes for the two surround directions (i.e., the direction-slope interaction was insignificant), which implies that the relationship between disparity tuning in the center and the surround was the same, regardless of the direction of motion in the surround. This translates to the slopes of the two relationships being parallel. A few neurons did show an interaction, however, and they are discussed later.

Cells with similar center and surround disparity tuning. Only four cells showed a significant, positive relationship between the disparity tuning in the center and the disparity tuning in the surround. Figure $9 D-F$ shows an example. In both the center $(D)$ and the surround $(E)$, responses tend to increase as disparities go 

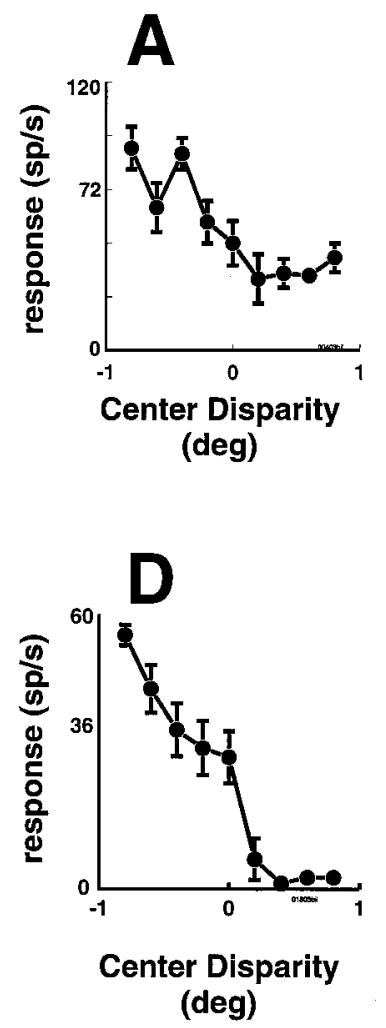
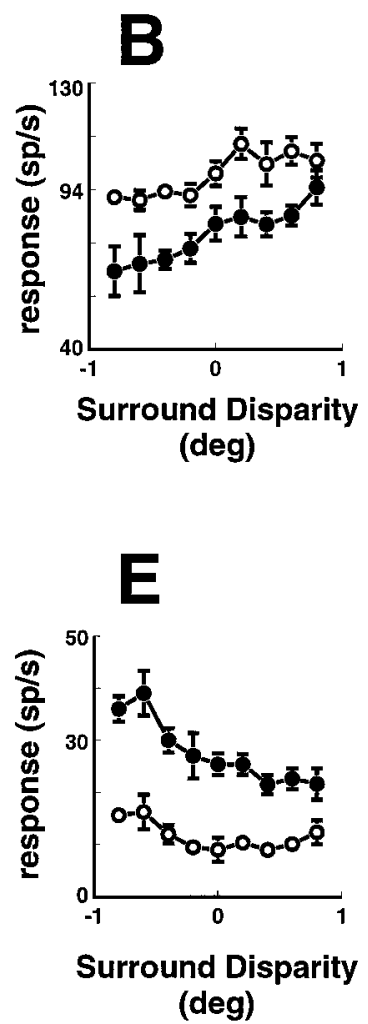

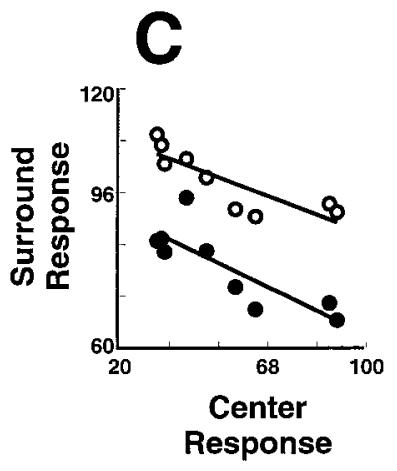

Negative Correlation

$\mathrm{n}=\mathbf{3 0}$

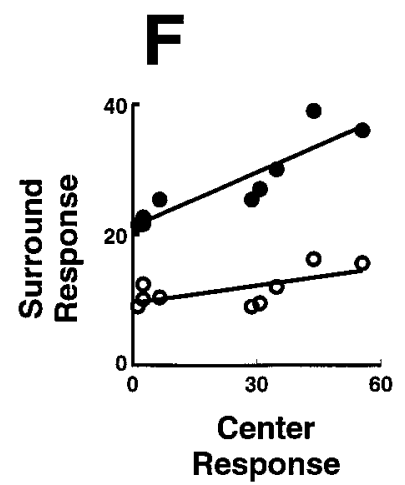

Positive Correlation

$\mathrm{n}=\mathbf{4}$

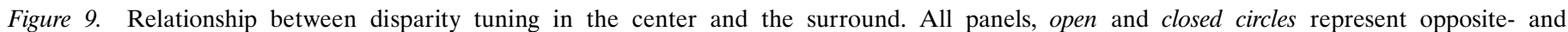

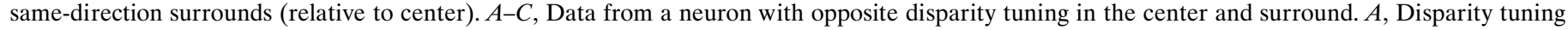

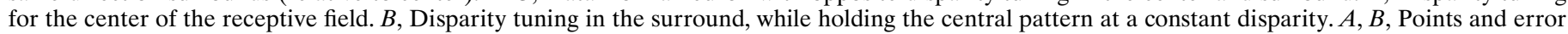

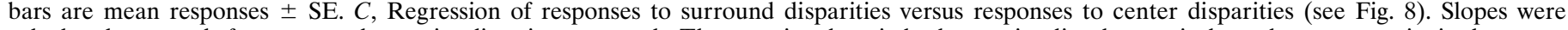

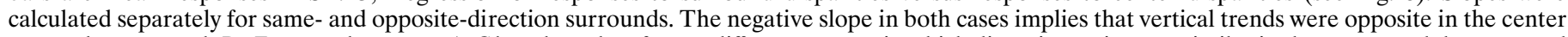

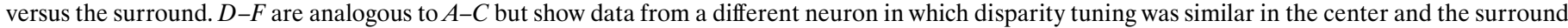
(thus positive slopes in $F$ ).

from negative to positive. The slope of the relationship between center and surround disparity tuning is thus positive $(F)$, reflecting the similarity of disparity effects in the center and the surround. Cells in this category, like the preceding category, did not show a significant direction-slope interaction, suggesting that the relationship between center and surround disparity tuning was the same for the two directions in the surround.

Cells with conditional (interactive) center and surround disparity tuning. Only 12 cells showed a significant direction-slope interaction, meaning that the slope of the relationship between disparity tuning in the center and disparity tuning in the surround was different for different directions in the surround. In twothirds (8/12) of these, the overall slope (the average of the individual slopes) was negative, implying that the inverse relationship between center and surround disparity tuning was generally preserved in this category of cells.

Cells with unrelated center and surround disparity tuning. The remaining $64 \%$ of the neurons $(81 / 127)$ did not show a significant relationship between disparity tuning in the center and disparity tuning in the surround. However, many of these were insensitive to disparity in the surround, and some were insensitive to disparity in the center, so testing the relationship between surround and center tuning was not meaningful.

If we limit ourselves to the 45 neurons showing significant disparity effects in the center and in the surround (based on oneand two-way ANOVAs, above), we find that only $36 \%$ (16/45) showed no relationship between center and surround disparity tuning; that is, two-thirds $(64 \%, 29 / 45)$ showed a significant relationship (20 negative, 0 positive, 9 interactive). Therefore, to the extent that MT receptive fields sense disparity, there tends to be an inverse relationship between disparity effects in the center and disparity effects in the surround.

Even these estimates are conservative, however, because our tests were predisposed to overlook significant relationships between center and surround disparity tuning. First, regression slopes were calculated with only nine points (corresponding to nine disparities), so the tests were inherently weak. Second, our tests assumed a linear relationship between center and surround tuning, and to the extent that the actual relationships were nonlinear, the power of our tests (the ability to detect significant slopes) was reduced. Third, regression assumes that independent variables (in this case the center response) are measured without error, and to the extent that error exists, as it did in our case, the power to detect significant slopes is reduced further. Finally, MT neurons respond best to large stimuli in the surround (Allman et al., 1985a,b). Because our patterns occupied only a small fraction $(5-10 \%)$ of the surround, we expect the effects of these patterns to be small (see also Discussion). This would further diminish our ability to detect significant center-surround relationships.

\section{Summary of center-surround interactions}

The two-way ANOVA discussed above confirms previous reports that MT responses are relatively high when center and surround patterns move in opposite directions and relatively low when they 


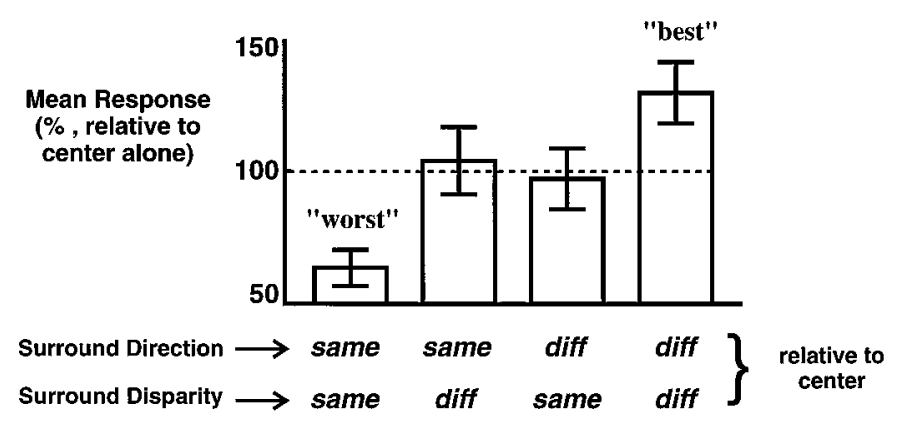

Figure 10. Pooled data from "additive" cells $(n=30)$. The dashed horizontal line represents the response to the central pattern by itself (normalized to be 100\%). Each bar shows mean normalized response, \pm $\mathrm{SE}$, for various combinations of direction and disparity. Responses were typically lowest (left bar) when center and surround patterns had different directions and disparities and highest (right bar) when both direction and disparity were different. The middle bars show that a difference in direction or disparity was sufficient to restore firing to the unsuppressed rate (i.e., not different from $100 \%$ of the center-alone response).

move in the same direction. Our multiple regression analysis further shows that responses are relatively high when center and surround stimuli have different, rather than equal, disparities. Finally, both the ANOVA and regression analyses suggest that direction and disparity effects are mainly additive; that is, each variable's effect tends to be independent of the other. On the basis of these findings, we can make a simple prediction, which is that responses should be weakest when center and surround stimuli have the same direction and disparity, and strongest when center and surround stimuli have different directions and disparities. In other words, the more the center and surround stimuli differ, in terms of motion and depth, the better in general these neurons should respond.

Figure 10 shows that this was indeed the case. The four bars in the figure represent mean responses ( $n=30$, additive cells only) to four basic types of stimulus: (1) the "worst" stimulus, in which center and surround patterns have the same direction and disparity; (2) the "best" stimulus, in which both direction and disparity are different; (3) an intermediate condition, in which the center and surround see different directions but the same disparity; and (4) an intermediate condition, in which the center and surround see the same direction but different disparities. Because nine disparities were tested, the definition of a "different" disparity in the surround is an arbitrary choice among the eight disparities not equal to the disparity in the center. We chose (among these eight) the disparity giving the strongest response; as such, this analysis represents a best-case scenario.

The mean responses plotted in Figure 10 are normalized on a cell-by-cell basis to the response to the center pattern by itself. Thus we see that the "worst" stimulus suppresses activity by $36 \%$, the intermediate stimuli give responses comparable to the center alone, and the "best" stimulus facilitates activity by $30 \%$. Therefore, to the extent that the center and surround see different directions and/or depths, MT responses tend to increase.

\section{DISCUSSION}

These experiments were performed to test the effects of disparity in the MT receptive field surround. MT neurons are known to be selective for disparity in their "classical" (central) receptive field (Maunsell and Van Essen, 1983b), but the effects of disparity in the surround have not been measured previously. We did so by showing surround patterns at variable disparity while holding a central pattern at a fixed disparity. In roughly half the neurons exhibiting a surround, responses were significantly modulated by disparity in the surround. Most of these were also affected by the direction of the surround patterns, and the direction and disparity effects were generally additive or independent. The magnitudes of the direction and disparity effects were comparable.

As we mentioned earlier, Allman et al. (1985a) showed that MT neurons tend to be suppressed by motion in the surround, but this suppression is relaxed when the speed or direction of surround motion is different from that in the center. Therefore, a requirement for strong activity is that receptive fields roughly coincide with discontinuities of the velocity field (note that the absence of motion in the surround represents a discontinuity when there is motion in the center). Such discontinuities are common, of course, near the edges of moving objects. Therefore, the velocity-antagonism of MT surrounds could act as a simple mechanism for locating image discontinuities (Allman et al., 1985b). This idea is supported by the present findings, which show that MT activities tend to increase as center and surround disparities become increasingly different. Again, this is consistent with an MT mechanism that heightens responses in the presence of image discontinuities.

It is important to detect such discontinuities, because we must be able to comprehend images that have several moving parts. Therefore, images must be divided into segments to be processed separately (Braddick, 1993; Stoner and Albright, 1993). These segments could be chosen arbitrarily, and image motion could be represented in terms of the average motion in each segment. However, if the segments are too large, more than one object could appear in a given segment, and the average motion in this segment would be meaningless. On the other hand, if the segments are too small, motion estimates become confounded with the orientation of edges [the aperture problem (Movshon et al., 1985)]. However, if each separately moving part of the image is defined as a segment, then the average motion in each segment is meaningful-it is the coherent movement of the object-and different segments would reflect the movement of separate objects. Therefore, object-based segmentation is a crucial part of motion processing.

Surrounds are not necessarily required for image segmentation. In situations in which our eyes are still and only a single object moves before us, the classical MT receptive field may be sufficient to calculate the object's velocity and identify where it is. MT receptive fields are topographically organized and small enough (typically with diameter $=$ distance to fovea) to give at least coarse information about stimulus location (Maunsell and Van Essen, 1983a). Therefore, the activity of a given neuron can be thought of as reflecting the probability that the stimulus location coincides with that neuron's receptive field position (and that the stimulus velocity matches that neurons' preferred velocity).

In many cases, however, the motion of an object may occur simultaneously with other motions on the retina. For example, several objects may be moving in different parts of the image, or the entire image may be moving (on the retina) because the eye is moving. Let us assume that an observer makes a smoothpursuit eye movement from right to left, either because he is tracking a moving object, or because he is moving while tracking a stationary object (Fig. 11). In that case the retina sees a rightward movement of the entire scene, which we will refer to as the "background." For simplicity, we will assume that the background is a large, frontoparallel surface.

Under these conditions, to detect the separate movement of the 


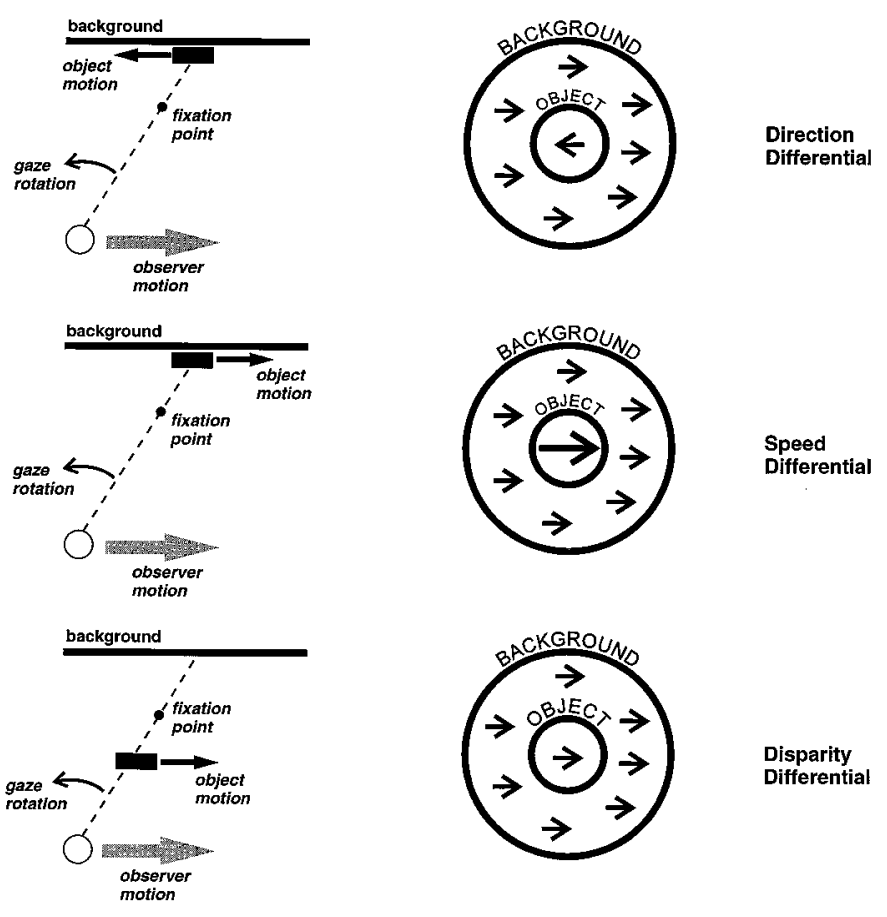

Figure 11. Hypothetical situation showing why surrounds must be antagonistic to the center in terms of direction, speed, and disparity to reliably detect object movement relative to background. An observer is assumed to be moving along a straight path while tracking a stationary object off to the left. This causes the background to move right across the retina. An object moving relative to background will always create a differential velocity on the retina, provided it is close to the background (top panels). If the object moves left with substantial speed (top panel), its retinal motion will be opposite in direction to the background motion. If it is moving right (middle panel), it will move in the same direction as the background but at a higher speed. However, if the object is in the foreground and moving right, its retinal velocity may match the retinal velocity of the background. In this case, its disparity must be different from the background, and this provides a center-surround differential with respect to depth. Because center-surround interactions in MT are direction-, speed-, and disparity-antagonistic, any of these three conditions is sufficient to "unsuppress" neurons with receptive fields centered on the moving object. In contrast, neurons that see background motion in both the center and the surround should remain suppressed.

object, we must distinguish its movement from that of the background. If the object is close to the background, then its motion relative to the background will produce a relative motion signal on the retina (Fig. 11). It was shown before that differential direction and speed cues between the center and the surround cause firing rates to increase (Allman et al., 1985b). Because there is likely to be a neuron whose classical field sees the object and whose surround sees the background, we can expect its activity to be strong because of the relative motion it sees between its center and surround. In contrast, neurons with classical receptive fields centered in other parts of the visual field would not see a motion differential and should be less activated (or more suppressed).

There are situations, however, where relative motion fails to distinguish an object from its background. If the object is in the foreground, its motion relative to background becomes confounded by a parallax effect, which makes it appear to move left with respect to the background (Fig. 11). If the object is actually moving right, it may move across the retina with the same velocity as the background. In this case all MT neurons would see the same velocity in their center and their surround; i.e., the relative motion cue would be lost. However, this problem can only arise if the object is at a different depth from the background-otherwise it could not be moving relative to the background and still appear to be moving at the same velocity. Neurons with center-surround antagonism based on disparity therefore could identify the object as separate from its background. This is precisely what our data reveal: even when surround motion has the same direction and speed as the center, a disparity differential is sufficient to substantially increase the firing rate (Fig. 10). Therefore, the disparity-based antagonism of the MT surround is a critical complement to the antagonism based on direction and speed. Allman et al. (1985b), in fact predicted antagonistic disparity tuning in the MT surround.

The scenario presented above is meant to demonstrate how surrounds that are antagonistic to the center in terms of direction, speed, and disparity cause MT firing rates to have predictive value about image discontinuities. However, two important simplifications were used. First, we tacitly assume that neurons seeing a center-surround discontinuity are "active," whereas neurons that see uniformity are "inactive." In reality, we would expect a distribution of activity whose shape encodes the locations of discontinuities. In this sense, it is again intuitive to think of each MT neuron's activity as reflecting the probability that the stimulus matches its velocity, depth, and spatial tuning.

The second simplification was that of a frontoparallel background. In natural scenes, the "background" (the bulk of the image) is commonly a ground plane, which is tilted with respect to the observer. In this case, different parts of the surround may see different directions, speeds, and disparities. As a result, it is possible that the stimulus could match the center in certain parts of the surround (in terms of velocity and depth) but differ from the center in other parts of the surround. If so, we might expect the surround stimulus to have an intermediate effect; i.e., somewhere between being the same as the center and different from the center. However, Orban and colleagues (Xiao et al., 1997) have found MT surrounds that are specifically tuned for speed gradients, which are associated with slanted surfaces. This suggests that MT neurons accomodate different types of background (e.g., frontoparallel vs slanted) by being specifically selective for them. In other words, center-surround comparisons could be made by different neurons under different conditions, depending on the structure of the stimulus that fills the surround. Future studies will be needed to understand how neurons respond to a slanted surround stimulus combined with a separate object moving through the center.

The preceding arguments should not be taken to mean that MT surrounds have a role only in segmentation. Work by Orban and colleagues (Xiao et al., 1997), in fact strongly suggests that these surrounds also play a role in computing surface orientation, and of course other functions are possible. Nevertheless, the available data clearly support the idea that at least one basic role of the MT surrounds involves scene segmentation. Specifically, (1) MT classical receptive fields are selective for direction, speed, and disparity; (2) MT surrounds are selective for direction, speed, and disparity; (3) all three center and surround selectivities are opposed, or antagonistic; and (4) the effects of direction and disparity are generally independent (the interactions between speed and direction/disparity effects have not been tested). An obvious conclusion is therefore that center-surround interactions in MT provide information about image differences between the center and the surround. 
The fourth point made above - that direction and disparity effects are independent-is important. For simplicity, let us assume that direction and disparity effects also combine linearly with speed. The significance of this linearity is that single-unit firing rates carry information about discontinuities without reflecting the specific cues that define them. A change in a neuron's response could mean that direction in the surround had changed (assuming a constant central stimulus) or that disparity had changed or both. Whatever the case, the change in response implies a difference between the center and the surround. On the other hand, if direction, speed, and disparity effects all depended on each other, the cell's firing rate would not have a straightforward meaning regarding the degree of similarity between the center and the surround. Therefore, the independence of the effects of direction and disparity (and assumedly speed) allows us to interpret activity as the likelihood that a difference exists between the center and the surround. Again, a simple differencing mechanism of this kind would be invaluable for segmenting an image.

In contrast to the studies of Allman et al. (1985b), our surround stimuli were composed of discrete patterns, rather than a uniform stimulus across the surround. The discrete patterns have the disadvantage that they cover a small part of the surround. Because MT surrounds exhibit spatial summation (Allman et al., 1985b), the magnitude of effects observed in our study are probably low estimates of the magnitudes one would see with larger, uniform surround stimuli.

These studies do not distinguish between absolute and relative disparity effects in the surround. That is, if the center pattern were presented at other (nonpreferred) disparities, it is not clear whether the disparity tuning in the surround would remain constant or vary with the disparity of the center. The latter would imply that individual cells encode relative depth between the center and surround. However, even if surround tuning is constant in each cell, information about relative depth could still be represented in the population by means of neuronal subsets tuned for different center-surround disparity combinations.

In summary, we have shown that center-surround interactions in the MT receptive field are modulated by binocular disparity. Like the effects of direction and speed (Allman et al., 1985a), the effects of disparity in the surround are antagonistic to the center; that is, cells are suppressed when the center and the surround are stimulated with the same disparity. A simple, unifying hypothesis concerning the effects of direction, speed, and disparity is that surrounds are part of a differencing mechanism that responds preferentially to image discontinuities. Given the size of MT receptive fields, this differencing mechanism is probably not involved in the precise mapping of object boundaries, but rather in computations that require a coarse parsing of the image into separately moving components.

\section{APPENDIX: STATISTICAL METHODS}

\section{Linear Models}

Most of the analyses done for this study were based on the general linear model, or GLM. This technique uses a regression framework to solve regression and ANOVA problems, as well as "mixed" problems that combine quantitative and discrete variables (Fox, 1997). Below we describe the different models used to analyze the data, followed by a discussion of the methods used for hypothesis testing.

\section{One-way ANOVA}

A simple one-way ANOVA was used to test the effects of disparity in the receptive field center. The GLM implementation of one-way ANOVA is given by:

$$
y=\mu+\alpha_{1} x_{1}+\alpha_{2} x_{2}+\ldots+\alpha_{8} x_{8} .
$$

The complete model contains one such equation for each data point. $y$ is an individual response measurement, $x_{1}-x_{8}$ are the independent variables, and $\alpha_{1}-\alpha_{8}$ are the parameters expressing the effect of each independent variable. $x_{1}-x_{8}$ are "dummy" variables, taking the value 0 or 1 , and are used to incorporate nominal scale (qualitative) data into the regression equation. $\mu$ is the offset parameter, representing the overall mean.

\section{Two-way ANOVA}

To simultaneously analyze direction and disparity effects in the surround, the GLM was configured as a two-way ANOVA, whose equation is:

$$
\begin{aligned}
y=\mu+\alpha x_{1}+\beta_{1} x_{2} & +\beta_{2} x_{3}+\ldots \\
& +\beta_{8} x_{9}+\gamma_{1} x_{1} x_{2}+\gamma_{2} x_{1} x_{3}+\ldots+\gamma_{8} x_{1} x_{9} .
\end{aligned}
$$

Here, $y$ is the response measurement, and $x_{1}-x_{9}$ are the independent variables. The $\alpha$ parameter represents the effect of direction, whereas $\beta$ represents the effects of disparity, and $\gamma$ represents the direction-disparity interaction. The three-way ANOVA is similar but has three main factors (as opposed to two) and three first-order interactions (as opposed to one).

\section{Comparison of disparity-tuning curves}

To compare the shape of two different disparity tuning curves, we expressed the responses under one condition as a function of the responses under the other condition. For example, to compare center and surround disparity tuning, we defined the independent variable as the response to a particular disparity in the center, and defined the dependent variable as the response to the same disparity in the surround. These definitions made it possible to study the center-surround relationship with regression techniques.

To compare disparity tuning curves under condition 1 versus condition 2 , the model equation is simply:

$$
y=\mu+\alpha x
$$

where $y$ is the response to a particular disparity under condition 1 (e.g., in the surround), and $x$ is the response to the same disparity under condition 2 (e.g., in the surround). $\alpha$ is the slope of the $x-y$ relationship, and $\mu$ is the intercept.

In one of our analyses, we studied the relationship between center and surround tuning - again, using linear regression-but here we included a dummy variable to represent the effect of direction. This allowed us to determine whether the centersurround relationship was itself dependent on the direction of motion in the surround. The model equation was:

$$
y=\mu+\alpha x_{1}+\beta x_{2}+\gamma x_{1} x_{2} .
$$

Here, $y$ is the response to a given disparity in the surround, and $x_{1}$ is the quantitative independent variable representing the response to the same disparity in the center. $x_{2}$ is a dummy variable indicating the direction of motion in the surround ( 0 or 1$)$.

This equation generates two linear curves, each representing the center-surround relationship for one of the two surround directions. $\alpha$ represents the mean slope of these two curves, 
whereas $\gamma$, the interaction parameter, is the difference in slope for the two different directions. $\beta$ is the difference between the intercepts of the two curves, and $\mu$ is the mean intercept.

\section{Hypothesis tests}

After we fit a given model (e.g., one- or two-way ANOVA) to the data using standard linear regression techniques, the significance of each factor (e.g., disparity) was evaluated with incremental $F$ tests; that is, coefficients corresponding to a certain factor were set to zero and the model refitted, and the increase in the residual sum of squares (the lack of fit) was tested for significance ( $F$ test). Interaction terms were fitted first; if they were significant, no further tests were done. If interactions were not significant, the "main effects" (e.g., direction and disparity) were tested. This approach adheres to the marginality principle, which states that hypothesis tests concerning main effects are invalid in the presence of interactions (Fox, 1997).

\section{REFERENCES}

aki Saito H, Yukie M, Tanaka K, Hikosaka K, Fukada Y, Iwai E (1986) Integration of direction signals of image motion in the superior temporal sulcus of the macaque monkey. J Neurosci 6:145-157.

Allman JM, Kaas JH (1971) A representation of the visual field in the caudal third of the middle temporal gyrus of the owl monkey (aotus trivirgatus). Brain Res 31:85-105.

Allman JM, Miezin F, McGuinness EL (1985a) Direction- and velocityspecific responses from beyond the classical receptive field in the middle temporal visual area (MT). Perception 14:105-126.

Allman JM, Miezin F, McGuinness EL (1985b) Stimulus-specific responses from beyond the classical receptive field: neurophysiological mechanisms for local-global comparisons in visual neurons. Ann Rev Neurosci 8:407-430.

Braddick O (1993) Segmentation versus integration in visual-motion processing. Trends Neurosci 16:263-268.

Bradley DC, Qian N, Andersen RA (1995) Integration of motion and stereopsis in middle temporal cortical area of macaques. Nature 373:609-611.

Bradley DC, Maxwell M, Andersen RA, Banks MS, Shenoy KV (1996) Mechanisms of heading perception in primate visual-cortex. Science 273:1544-1547.

Bradley DC, Chang G, Andersen RA (1998) Encoding of 3D structurefrom-motion by primate area MT neurons. Nature 392:714-717.

Carmer SG, Swanson MR (1973) An evaluation of ten pairwise multiple comparison procedures by monte carlo methods. J Am Stat Assoc 68:66-74.

Colby CL, Duhamel JR, Goldberg ME (1993) Ventral intraparietal area of the macaque: anatomic location and visual response properties. J Neurophysiol 69:902-914.

Desimone R, Ungerleider L (1986) Multiple visual areas in the caudal superior temporal sulcus of the macaque. J Comp Neurol 248:164-189.

Dow BM (1974) Functional classes of cells and their laminar distribution in monkey visual cortex. J Neurophysiol 37:927-946.
Dubner R, Zeki SM (1971) Response properties and receptive fields of cells in an anatomically defined region of the superior temporal sulcus. Brain Res 35:528-532.

Fox J (1997) Applied regression analysis, linear models, and related methods. Thousand Oaks, CA: Sage Publications.

Gibson JJ (1950) Perception of the visual world. Boston: Houghton Mifflin.

Judge S, Richmond B, Chu F (1980) Implementation of magnetic search coils for measurement of eye position: an improved method. Vision Res 20:535-538.

Maunsell JH, Van Essen DC (1983a) Functional properties of neurons in middle temporal visual area of the macaque monkey. i. Selectivity for stimulus direction, orientation and speed. J Neurophysiol 49:1127-1147.

Maunsell JH, Van Essen DC (1983b) Functional properties of neurons in middle temporal visual area of the macaque monkey. ii. Binocular interactions and sensitivity to binocular disparity. J Neurophysiol 49:1148-1167.

Movshon JA, Adelson EH, Gizzi MS, Newsome WT (1985) The analysis of moving visual patterns. In: Study group on pattern recognition mechanisms (Chagas C, Gattass R, Gross CG, eds), pp 117-151. Vatican City: Pontifica Academia Scientiarium.

Nakayama K (1985) Biological image motion processing: a review. Vision Res 25:625-660.

Newsome WT, Wurtz RH (1988) Probing visual cortical function with discrete chemical lesions. Trends Neurosci 11:394-400.

Newsome WT, Britten KH, Movshon JA (1989) Neuronal correlates of a perceptual decision. Nature 341:52-54.

Schiller PH, Lee K (1994) The effects of lateral geniculate nucleus, area $\mathrm{v} 4$, and middle temporal (MT) lesions on visually guided eye movements. Vis Neurosci 11:229-241.

Siegel RM, Andersen RA (1986) Motion perceptual deficits following ibotenic acid lesions of the middle temporal area in the behaving rhesus monkey. Soc Neurosci Abstr 12:1183.

Snowden RJ, Treue S, Erickson RG, Andersen RA (1991) The response of area MT and V1 neurons to transparent motion. J Neurosci 11:2768-2785.

Stoner GR, Albright TD (1993) Image segmentation cues in motion processing: implications for modularity in vision. J Comp Neurol 5:129-149.

Tootell RBH, Reppas JB, Dale AM, Look RB, Sereno MI (1995) Visual-motion aftereffect in human cortical area MT revealed by functional magnetic-resonance-imaging. Nature 375:139-141.

Wallach H, O'Connell DN (1953) The kinetic depth effect. J Exp Psychol 45:205-217.

Warren WH (1995) Self-motion: visual perception and visual control. In: Perception of space and motion, Ch 8, pp 263-325. Los Angeles: Academic.

Xiao DK, Marcar VL, Raiguel SE, Orban GA (1997) Selectivity of macaque MT/V5 neurons for surface orientation in-depth specified by motion. Eur J Neurosci 9:956-964.

Zeki SM (1974) Functional organization of a visual area in the posterior bank of the superior temporal sulcus of the rhesus monkey. J Physiol (Lond) 236:549-573. 